\title{
X-ray nuclear emission of a sample of LINER Galaxies
}

\author{
O. González-Martín ${ }^{1} \dagger$, J. Masegosa ${ }^{1} \&$ I. Márquez ${ }^{1}$ \\ ${ }^{1}$ Instituto de Astrofísica de Andalucía (CSIC), Apdo 3004, 10080 Granada, Spain
}

\begin{abstract}
We report the results from an homogeneous analysis of the X-ray (ACIS-S/Chandra) data available for a sample of 52 LINER galaxies. The X-ray morphology has been classified attending to their nuclear compactness in the hard band (4.5-8.0 keV), into 2 categories: AGN-like nuclei (with a clearly identified unresolved nuclear source) and Starburst-like nuclei (without a clear nuclear source). $60 \%$ of the total sample are classified as AGNs, with a median luminosity of $\mathrm{L}_{\mathrm{X}}(2.0-10.0 \mathrm{keV})=2.5 \times 10^{40} \mathrm{erg} \mathrm{s}^{-1}$, which is an order of magnitude higher than that for SB-like nuclei. The spectral fitting allows to conclude that most of the objects need a non-negligible thermal contribution. When no spectral fitting can be performed (low signal-tonoise ratio), the Color-Color diagrams allow us to compute physical parameters such as density column, temperature of the thermal model or spectral index and therefore to analyze the origin of the X-ray emission. All X-ray morphology, spectral fitting and Color-Color diagrams allow conclude that a high percentage of LINER galaxies host AGN nuclei.
\end{abstract}

Keywords. galaxies, AGN, LINER, X-ray, Chandra.

\section{Introduction \& Sample}

LINERs are very common in the nearby universe. Pioneering works already estimate that at least $1 / 3$ of all the spiral galaxies are LINERs (Heckman et al. 1980). More than two decades after they were clasified, there is still an ongoing strong debate on the origin of the energy source in LINERs, with two main alternatives for the ionizing source being explored: either it is a low luminosity AGN (Filippenko \& Halpern, 1984), or it is of thermal origin from massive star formation (Filippenko \& Terlevich, 1992) and/or from shock heating mechanisms resulting from the massive stars evolution (Fosbury et al. 1978 and Dopita 1976). The search for a compact X-Ray nucleus in LINERs is indeed one of the most convincing evidences about their AGN nature. The excellent resolution of Chandra allows an investigation of the X-Ray nuclear properties of these galaxies.

All the 476 LINER galaxies in the compilation by Carrillo et al. (1999) have been searched by coordinates in Chandra archives, with 137 galaxies having public ACIS data. To minimize any bias due to incompleteness, only the 65 objects with high enough exposure times and therefore high $\mathrm{S} / \mathrm{N}$ ratio have been selected ( $\mathrm{t}>10000$ seconds). The final sample, after optical re-identification as LINER nuclei, comprises 52 galaxies.

\section{Data process}

The data products were analyzed in an uniform, self-consistent, manner using $C X C$ Chandra Interactive Analysis of Observations (CIAO $\ddagger$ ) software version 3.1. The spectral analysis was done with XSPEC (version 11.3.2). Level 2 event data from ACIS

$\dagger$ e-mail address: omaira@iaa.es

$\ddagger$ See http://asc.harvard.edu/ciao

I See http://cxc.heasarc.gsfc.nasa.gov/docs/xanadu/xspec/ 
instrument have been extracted from Chandra archive. Time intervals with high background levels have been excluded, using task $l c_{-}$clean.sl $\dagger \dagger$ in source-free sky regions of the same observation.

\section{Spectral fitting}

For the source selection, we made use of the nuclear positions from NVSS and 2MASS data base. Nuclear spectra were extracted using regions defined to include as many photons coming from the source as possible, but at the same time minimizing contamination from nearby sources and background. The background region was defined as either a source-free circular annulus or several circles surrounding each source, in order to take into account the spatial variations of the diffuse emission and to minimize effects related to the spatial variation of the CCD response. In order to use the $\chi^{2}$ statistic, the data were grouped to include at least 20 counts per spectral bin, before background substraction. For the spectral fitting any events with energies above $10.0 \mathrm{keV}$ or below $0.5 \mathrm{keV}$ are excluded.

The spectra in the $0.5-10.0 \mathrm{keV}$ passband were modelled with a single [MEKAL (ME), Raymond-Smith (RS) or Power Law (PL)] component first and second with a two component $[\mathrm{ME}+\mathrm{PL}$ or RS+PL] model. Single models are representative for sources dominated either by thermal emission (such as Supernova Remnants and galactic bubles), or non-thermal emission (such as accreting compact objects), and two component models correspond to composite objects. Note that number counts were insufficient to employ detailed spectral fitting in 28 out of 52 objects.

Attending to the spectral fittings the use of two components models better reproduce the X-ray energy distribution of most of these 24 objects, indicating a non-negligible non-thermal contribution in our sample.

\section{Luminosities}

The luminosities and fluxes of the individual nuclear sources have been computed based on the best-fit model for the 24 galaxies abovefł.

We have done an empirical calibration from these 24 objects with high signal-to-noise ratio from flux estimation using PIMMS $\boldsymbol{\top}$ assuming an intrinsic power law slope of 1.8, corrected for Galactic absorption $\left(\mathrm{N}_{\mathrm{H}}=3 \times 10^{20} \mathrm{~cm}^{-2}\right)$. In Fig. 1 (left) our estimated luminosity is plotted versus the value obtained from the direct integration of the spectral energy distribution. The luminosities are well correlated within a factor of three of the real one. The 2.0-10.0 keV luminosities are therefore provided for the whole sample, using the spectral energy distribution fit, when it is available, and from this calibration otherwise.

\section{Morphological Classification}

Since we focus our attention in the nuclear sources, no attempt has been made to fully characterize the flux and the spectral properties (when possible) of the extranuclear sources whose study is out of the scope of the paper. We have classified the nuclear morphology attending to the compactness in the hard band (4.5 to $8.0 \mathrm{keV}$ ). Therefore the sample has been grouped into 2 main categories:

$\dagger \dagger$ See http://cxc.harvard.edu/ciao/download/scripts/

$\ddagger \ddagger$ Throughout this paper we use a cosmological constant of $\mathrm{H}_{\mathrm{o}}=75 \mathrm{~km} \mathrm{~s}^{-1} \mathrm{Mpc}^{-1}$

ๆๆ See http://heasarc.gsfc.nasa.gov/Tools/w3pimms.html 

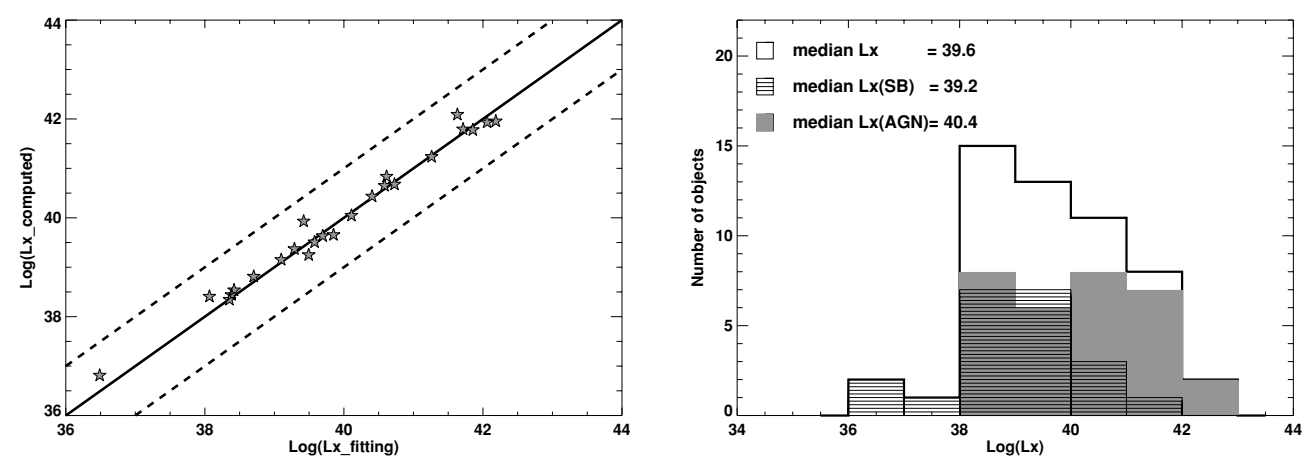

Figure 1. (left): Luminosity estimation versus real luminosity. Dashed-lines are 10 times above and below real value, respectively and continuous line indicates the best luminosity estimation. (right): Luminosity histogram for the whole sample (empty histogram), Objects clasified as AGN-like (grey histogram) and Starburst -like objects (dashed histogram).

- AGN-like nuclei: We include all the galaxies with a clearly identified unresolved nuclear source in the hard band. 59.6\% (31/52) has been clasified as AGN-like nuclei in our sample and their median luminosity is $\mathrm{L}_{\mathrm{X}}(2-10 \mathrm{keV})=2.5 \times 10^{40} \mathrm{erg} \mathrm{s}^{-1}$ (Fig. 1, right).

- Starburst-like nuclei (SBs): Here we include all the objects without a clear nuclear source in the hard band. $40.4 \%$ of the sample of LINERs fall in this classification and their median luminosity is $\mathrm{L}_{\mathrm{X}}(2-10 \mathrm{keV})=1.0 \times 10^{39} \mathrm{erg} \mathrm{s}^{-1}$ (Fig. 1, right).

\section{Color-Color Diagrams}

The X-ray colors have also been studied for all the nuclear sources in our sample. Colors have been defined as the ratio of counts observed in the following energy bands: 0.6 to $0.9,0.9$ to $1.2,1.2$ to $1.6,1.6$ to $2.0,2.0$ to 4.5 , and 4.5 to $8.0 \mathrm{keV}$. The bands were chosen in order to maximize the detection as well as to obtain a good characterization of the spectra. In the last energy band, the range from 6.0 to $7.0 \mathrm{keV}$ has been excluded to avoid the possible contamination due to the FeK emission line. Therefore, 3 colors has been defined $\left(\mathrm{Q}_{\mathrm{A}}, \mathrm{Q}_{\mathrm{B}}\right.$ and $\left.\mathrm{Q}_{\mathrm{C}}\right)$ as $\mathrm{Q}=($ Hard-Soft $) /($ Hard+Soft $)$. Synthetic colors have been computed for PL, RS and PL+RS models by using PIMMS (see Fig. 2).

We have only considered the data with error less than $30 \%$. In the less energetic diagram $\left(\mathrm{Q}_{\mathrm{A}}\right.$ versus $\mathrm{Q}_{\mathrm{B}}$, Fig. 2, left) there are only a few objects highly obscured. Note that these objects has been clasified as AGN-like objects. Most of the objects are in the combination grid with low column density, where AGN and SB like nuclei appear together.

A handful of objects lie quite far of any grid; most of these objects have been studied in the literature as hosting a radio-jet, an additional contribution that has not been taken into a account in our fitting. In the $\mathrm{Q}_{\mathrm{B}}$ versus $\mathrm{Q}_{\mathrm{C}}$ plot (Fig. 2, centre) we can see that $\mathrm{Q}_{\mathrm{C}}$ is a good AGN activity estimator. However, not only a few objects classified as SB-like have a high $\mathrm{Q}_{\mathrm{C}}$, but also most of the objects classified as SB are not in the thermal model grid. This might be indicative that an additional non-thermal mechanism is needed to fit the spectral energy distribution.

Therefore, the use of Color-Color diagrams allows to also analyze the properties of the nuclear sources for which the spectral fitting is not possible. 

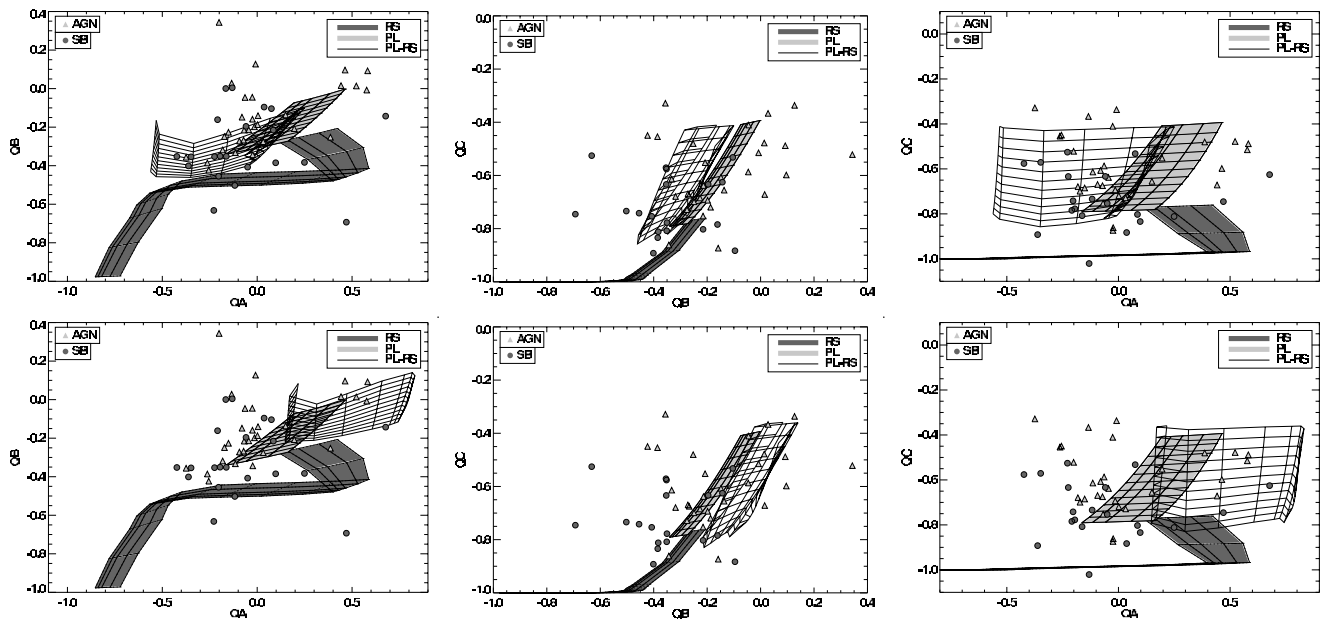

Figure 2. Color-color diagrams for a RS model (dark grey filled grid), PL model (light grey filled grid) and combinated model (empty grid) for $\mathrm{N}_{\mathrm{H}}=10^{20} \mathrm{~cm}^{-2}$ (top) and $\mathrm{N}_{\mathrm{H}}=10^{22} \mathrm{~cm}^{-2}$ (bottom). QB versus QA (left), QC versus QB (centre) and QC versus QA (right). Light-grey triangles are AGN-like objects and dark-grey circles are SB-like objects. The grids were calculated for $\Gamma=0.4$ to 2.6 for $\mathrm{PL}$ model, for $\mathrm{kT}=0.4$ to $4.0 \mathrm{keV}$ for the $\mathrm{RS}$ model and $\mathrm{N}_{\mathrm{H}}=(0.5-30.) \times 10^{20} \mathrm{~cm}^{-2}$ in the single model case.

\section{Conclusions}

In this paper we have described the detailed analysis of the X-ray spectral properties of the nuclear sources in an optically classified LINER galaxy sample, observed with Chandra ACIS-S. Our findings are summarized below:

- Morphologically, $60 \%$ of LINERs have been classified as AGN-like candidates, with median luminosity 10 times higher than that of SB-like objects.

- Both thermal and non-thermal contributions are required for the spectral fitting of most of the objects. Color-Color diagrams have confirmed this result.

- An empirical calibration for estimating X-ray luminosities $\left(\mathrm{L}_{\mathrm{X}}(2.0-10.0 \mathrm{keV})\right)$ has been done based on total counts, which allows a reliable estimation when the spectral fitting is not possible.

- Color-Color diagrams are a valid tool to estimate physical parameters, specially interesting to be used when the spectral fit is not possible.

\section{References}

Carrillo, R., Masegosa, J., Dultzin-Hacyan, D., \& Ordoñez, 1999, Rev. Mex. A\&A, 35, 18

Dopita, M. A., 1976 ApJ, 209, 395

Filippenko, A. V. \& Halpern, J. P. 1984 ApJ, 285, 458

Filippenko, A. V. \& Terlevich, R. 1992 ApJ, 397, L79

Fosbury, R. A. E., Mebold, U., Goss, W. M., \& Dopita, M. A. 1978 MNRAS, 183, 549

Heckman, T. M., Crane, P. C., \& Balick, B., 1980, A\&AS, 40, 295

Ho, L. C., Feigelson, E. D., Townsley, L. K., Sambruna, R. M., Garmire, G. P., Brandt, W. N., Filippenko, A. V., Griffiths, R. E., Ptak, A. F., \& Sargent W. L. W., 1985, ApJ, 549, L51 


\section{Discussion}

ELVIS: Is your definition of starburst based just on seeing extended X-ray emission? If so it is important to note that some extended X-ray emission is related to the nucleus as e.g. in NGC4151.

Gonzalez-Martin: Our starburst definition is only based on the non existence of a point-like source in the hard band $(4.5-8.0 \mathrm{KeV})$.

MEuRs: Some of your LINER sample may also have been investigated with ISO for the IR-based AGN versus starburst separation. If there is such overlap, to what degree do your results agree with the IR diagnostics?

Gonzalez-Martin: It is difficult to draw conclusion based in the present sample. Since our ACIS sample has been taken from the archive, when you compare with ISO data few objects have been observed. Therefore any conclusion based in the comparison between ISO and Chandra data need to be taken very carefully due to the bias from the way they have been selected.

MACCARONE: Have you looked at the radio properties of these objects to see if e.g. the radio to X-ray flux ratios or radio spectral indices are systematically different between AGN dominated LINERs and starburst dominated LINERs?

GonZALEZ-Martin: We haven't yet had a chance to look at the radio data. 